PROCEEDINGS OF THE

AMERICAN MATHEMATICAL SOCIETY

Volume 137, Number 9, September 2009, Pages 3091-3097

S 0002-9939(09)09859-1

Article electronically published on March 11, 2009

\title{
REDUCING SUBSPACES FOR A CLASS OF MULTIPLICATION OPERATORS ON THE DIRICHLET SPACE
}

\author{
LIANKUO ZHAO
}

(Communicated by Nigel J. Kalton)

\begin{abstract}
In this paper, we discuss reducing subspaces of multiplication operators $M_{\phi}$ on the Dirichlet space $\mathcal{D}$ defined by a Blaschke product $\phi$ with two zeros $a, b$ in the unit disk $\mathbb{D}$ and show that when $a+b=0, M_{\phi}$ has two proper ones; otherwise it has none. This is different from the cases of the Hardy space and the Bergman space.
\end{abstract}

\section{INTRODUCTION}

Let $\mathbb{D}$ be the open unit disk, and let $d A$ denote the normalized Lebesgue area measure on $\mathbb{D}$. The Dirichlet space $\mathcal{D}$ consists of an analytic function $f$ on $\mathbb{D}$ with finite Dirichlet integral

$$
D(f)=\int_{\mathbb{D}}\left|f^{\prime}\right|^{2} d A<\infty .
$$

Endow $\mathcal{D}$ with the norm $\|f\|$,

$$
\|f\|^{2}=|f(0)|^{2}+D(f) .
$$

Then $\mathcal{D}$ is a Hilbert space with inner product

$$
\langle f, g\rangle=f(0) \overline{g(0)}+\int_{\mathbb{D}} f^{\prime}(z) \overline{g^{\prime}(z)} d A(z)
$$

for $f, g \in \mathcal{D}$.

It is well known that $\mathcal{D}$ is a reproducing function space with reproducing kernel

$$
K_{\lambda}(z)=1+\log \frac{1}{1-\bar{\lambda} z}, \quad \lambda, z \in \mathbb{D},
$$

and for $f \in \mathcal{D}$,

$$
\left\langle f, K_{\lambda}\right\rangle=f(\lambda) .
$$

In recent years, the Dirichlet space has received a lot attention from the analytists. We refer readers to the survey paper 8 for more information about the Dirichlet space.

A reducing subspace $\mathcal{S}$ for an operator $A$ on a Hilbert space $\mathcal{H}$ is a closed subspace of $\mathcal{H}$ such that $A \mathcal{S} \subset \mathcal{S}$ and $A \mathcal{S}^{\perp} \subset \mathcal{S}^{\perp}$. The commutant of $A$, denoted $\{A\}^{\prime}$, is the (weak-star closed) algebra of bounded operators $B$ on $\mathcal{H}$ such that $B A=A B$. It is well known that $\mathcal{S}$ is a reducing subspace for $A$ if and only if $A P=P A$, where

Received by the editors June 25, 2008, and, in revised form, December 17, 2008.

2000 Mathematics Subject Classification. Primary 47A15, 46E22; Secondary 47S99.

Key words and phrases. Reducing subspace, multiplication operator, Dirichlet space.

(C)2009 American Mathematical Society 
$P$ is the orthogonal projection from $\mathcal{H}$ to $\mathcal{S}$. Thus the problem of characterizing reducing subspaces for an operator is closely related to the problem of describing its commutant.

In 9], a complete description of the reducing subspaces of weighted unilateral shift operators of finite multiplicity is obtained. Consequently, it is easy to verify that the multiplication operator $M_{z^{N}}$ induced by $z^{N}, N>1$ on the Dirichlet space and on the Bergman space has exact $2^{N}-2$ proper reducing subspaces; in particular, $M_{z^{2}}$ has just two proper ones. For the Bergman space, in [10] and [12], the authors used different methods, respectively, to show that any multiplication operator defined by a Blaschke product with two zeros in $\mathbb{D}$ has only two proper reducing subspaces. In 4, 5, 7, the authors deeply studied such problems for multiplication operators defined by finite Blaschke products. For the Hardy space, the reducing subspaces of the multiplication operators defined by inner functions are studied in [1, 6], since such operators are pure isometry.

In this paper, we consider the reducing subspaces of multiplication operators on the Dirichlet space defined by Blaschke products with two zeros in $\mathbb{D}$. Note that our method is different from those in [10] and [12]. We fix some notation to state the result.

Recall that a function $\phi$ is called a multiplier of $\mathcal{D}$ if $\phi \mathcal{D} \subset \mathcal{D}$. Let $\mathcal{M}$ denote the multipliers of $\mathcal{D}$. An easy application of the closed graph theorem shows that the multiplication operator $M_{\phi}: f \rightarrow \phi f$ defined by a multiplier $\phi$ on $\mathcal{D}$ is a bounded linear operator. Moreover multipliers are bounded analytic functions on $\mathbb{D}$, and every finite Blaschke product is a multiplier for $\mathcal{D}$.

Let $\varphi_{\lambda}(z)=\frac{\lambda-z}{1-\lambda z}, \lambda, z \in \mathbb{D}$, be the Möbius transform.

Now we state the main result of the paper.

Theorem 1.1. Let $\phi=\varphi_{a} \varphi_{b}$ be a Blaschke product with two zeros $a$ and $b$ in $\mathbb{D}$, and let $M_{\phi}: \mathcal{D} \rightarrow \mathcal{D}$ be the multiplication operator defined by $\phi$. If $a+b=0$, then $M_{\phi}$ has only two proper reducing subspaces. If $a+b \neq 0$, then $M_{\phi}$ has no proper reducing subspaces.

As an application, we describe the multiplication operators on $\mathcal{D}$ defined by multipliers which are unitarily equivalent to $M_{z^{2}}$, and we show that such multipliers are a constant multiple of $z^{2}$. We have the following consequence.

Corollary 1.2. Let $\phi \in \mathcal{M}$. Then $M_{\phi}$ is unitarily equivalent to $M_{z^{2}}$ if and only if $\phi(z)=\lambda z^{2}$ for some $\lambda$ with $|\lambda|=1$.

\section{Proof of the main Result AND its APPliCAtion}

In this section, we give the proof of the main result and one application as mentioned in the introduction.

Since reducing subspaces of an operator corresponds to orthogonal projections in its commutant, firstly, we provide a discussion on the commutant of $M_{\phi}$ for $\phi \in \mathcal{M}$, which may be known by many people; similar results for the Hardy space and the Bergman space appeared in [3, 11.

Lemma 2.1. Let $\phi \in \mathcal{M}$. Then $T \in\left\{M_{\phi}\right\}^{\prime}$ if and only if for any $\alpha \in \mathbb{D}, T^{*} K_{\alpha} \perp$ $(\phi-\phi(\alpha)) \mathcal{D}$.

Proof. Suppose first that $T \in\left\{M_{\phi}\right\}^{\prime}$. Then for any $f \in \mathcal{D}$,

$$
\left\langle T^{*} K_{\alpha},(\phi-\phi(\alpha)) f\right\rangle=\left\langle K_{\alpha}, T(\phi-\phi(\alpha)) f\right\rangle=\left\langle K_{\alpha},(\phi-\phi(\alpha)) T f\right\rangle=0 .
$$


Now suppose that for any $\alpha \in \mathbb{D}, T^{*} K_{\alpha} \perp(\phi-\phi(\alpha)) \mathcal{D}$. Then for any $f \in \mathcal{D}$, $\left\langle\left(M_{\phi} T-T M_{\phi}\right) f, K_{\alpha}\right\rangle=\left\langle\phi T f, K_{\alpha}\right\rangle-\left\langle\phi f, T^{*} K_{\alpha}\right\rangle=\left\langle(\phi(\alpha)-\phi) f, T^{*} K_{\alpha}\right\rangle=0$.

Therefore for any $\alpha \in \mathbb{D},\left(\left(M_{\phi} T-T M_{\phi}\right) f\right)(\alpha)=0$, and hence $T \in\left\{M_{\phi}\right\}^{\prime}$.

Lemma 2.2. Let $\phi=\varphi_{a} \varphi_{b}, \lambda=\frac{a+b-|a|^{2} b-|b|^{2} a}{1-|a|^{2}|b|^{2}}, \psi(z)=z \varphi_{\lambda}(z)$. Then $\left\{M_{\phi}\right\}^{\prime}=$ $\left\{M_{\psi}\right\}^{\prime}$.

Proof. A direct computation shows that $\psi=\varphi_{a b} \circ \phi$ and $\phi=\varphi_{a b} \circ \psi$. Thus in order to prove the lemma, it is enough to prove $\left\{M_{\phi}\right\}^{\prime} \subset\left\{M_{\psi}\right\}^{\prime}$.

By Lemma 2.1, for any $T \in\left\{M_{\phi}\right\}^{\prime}$ and $\alpha \in \mathbb{D}, T^{*} K_{\alpha} \perp(\phi-\phi(\alpha)) \mathcal{D}$. Since $\psi-\psi(\alpha)=\frac{\varphi_{a b} \circ \phi-\varphi_{a b}(\phi(\alpha))}{\phi-\phi(\alpha)}(\phi-\phi(\alpha))$ and $\frac{\varphi_{a b} \circ \phi-\varphi_{a b}(\phi(\alpha))}{\phi-\phi(\alpha)} \in \mathcal{M}$,

$$
(\psi-\psi(\alpha)) \mathcal{D} \subset(\phi-\phi(\alpha)) \mathcal{D} \text {. }
$$

It follows that $T^{*} K_{\alpha} \perp(\psi-\psi(\alpha)) \mathcal{D}$. By Lemma 2.1, $T \in\left\{M_{\psi}\right\}^{\prime}$.

To prove Theorem 1.1, by Lemma 2.2, it is enough to describe the reducing subspaces of $M_{\psi}$ with $\psi(z)=z \varphi_{\lambda}(z), \lambda \in \mathbb{D}$.

To continue, we need a representation formula for the Dirichlet integral given by Carleson in [2].

Let $f=B S F$ be the canonical factorization of $f$, where $B=\prod_{j=1}^{\infty} \frac{\overline{a_{j}}}{\left|a_{j}\right|} \frac{a_{j}-z}{1-\overline{a_{j} z}}$ is a Blaschke product, $S$ is the singular part of $f, \mu$ is the corresponding singular measure, and $F$ is the outer part of $f$. Then

$$
\begin{aligned}
\pi D(f)= & \int_{\mathbb{T}} \sum_{n=1}^{\infty} P_{\alpha_{n}}(\xi)|f(\xi)|^{2} \frac{|d \xi|}{2 \pi}+\int_{\mathbb{T}} \int_{\mathbb{T}} \frac{2}{|\zeta-\xi|^{2}}|f(\xi)|^{2} d \mu(\zeta) \frac{|d \xi|}{2 \pi} \\
& +\int_{\mathbb{T}} \int_{\mathbb{T}} \frac{\left(e^{2 u(\zeta)}-e^{2 u(\xi)}\right)(u(\zeta)-u(\xi))}{|\zeta-\xi|^{2}} \frac{|d \zeta|}{2 \pi} \frac{|d \xi|}{2 \pi}
\end{aligned}
$$

for $f \in \mathcal{D}$, where $u(\xi)=\log |f(\xi)|$, and $P_{\alpha}(\xi)$ is the Poisson kernel.

Now, we present the proof of the main result.

Proof of Theorem 1.1. Let $\lambda=\frac{a+b-|a|^{2} b-|b|^{2} a}{1-|a|^{2}|b|^{2}}$. We will prove the theorem in two cases.

Case A. $a+b=0$. Then $\lambda=0$. By Lemma 2.2, $M_{\phi}$ and $M_{z^{2}}$ have the same reducing subspaces. As mentioned in the introduction, Theorem B in 9] implies that $M_{z^{2}}$ has only two proper reducing subspaces

$$
X_{n}=\overline{\operatorname{Span}}\left\{z^{n+2 k}: k=0,1,2, \cdots\right\}, \quad n=0,1 .
$$

Case B. $a+b \neq 0$. Then $\lambda \neq 0$. By Lemma 2.2, $M_{\phi}$ and $M_{\psi}$ have the same reducing subspaces, where $\psi(z)=z \varphi_{\lambda}(z)$.

Assume that $X$ is a proper reducing subspace of $M_{\psi}$. Then $X^{\perp}$ is also a proper reducing subspace of $M_{\psi}$.

For every $f \in \mathcal{D}, f$ has decomposition $f=f_{1}+f_{2}$ with $f_{1} \in X$ and $f_{2} \in X^{\perp}$. Then for any $g \in X \ominus \psi X$, we have

$$
\langle g, \psi f\rangle=\left\langle g, \psi f_{1}\right\rangle+\left\langle g, \psi f_{2}\right\rangle=0,
$$

since both $X$ and $X^{\perp}$ are reducing subspaces of $M_{\psi}$. Hence

$$
X \ominus \psi X \subset \mathcal{D} \ominus \psi \mathcal{D} .
$$


Similarly

$$
X^{\perp} \ominus \psi X^{\perp} \subset \mathcal{D} \ominus \psi \mathcal{D} .
$$

Again, since $X, X^{\perp}$ are reducing subspaces of $M_{\psi}$, for any nonzero

$$
f \in X \ominus \psi X, \quad g \in X^{\perp} \ominus \psi X^{\perp}
$$

and $n \geq 0$,

$$
\left\langle\psi^{n} f, \psi^{n} g\right\rangle=0
$$

Since $\psi(0)=0$, for $n \geq 1, k=0,1,2,3$, we have

$$
\left\|\psi^{n}\left(f+i^{k} g\right)\right\|^{2}=D\left(\psi^{n}\left(f+i^{k} g\right)\right),
$$

where $i$ is the imaginary unit.

By Carleson's integral formula,

$$
\begin{aligned}
& D\left(\psi^{n}\left(f+i^{k} g\right)\right)=\frac{n}{\pi} \int_{\mathbb{T}}\left(\left(1+P_{\lambda}(\xi)\right)\left|f(\xi)+i^{k} g(\xi)\right|^{2}\right) \frac{|d \xi|}{2 \pi}+D\left(f+i^{k} g\right) \\
= & \frac{n}{\pi} \int_{\mathbb{T}}\left(\left(1+P_{\lambda}(\xi)\right)\left|f(\xi)+i^{k} g(\xi)\right|^{2}\right) \frac{|d \xi|}{2 \pi}+\left\|f+i^{k} g\right\|^{2}-\left|f(0)+i^{k} g(0)\right|^{2} .
\end{aligned}
$$

Hence, by the polarization identity,

$$
\begin{aligned}
0 & =\left\langle\psi^{n} f, \psi^{n} g\right\rangle=\sum_{k=0}^{3} \frac{i^{k}}{4}\left\|\psi^{n}\left(f+i^{k} g\right)\right\|^{2} \\
& =\frac{n}{\pi} \int_{\mathbb{T}}\left(1+P_{\lambda}(\xi)\right) f(\xi) \overline{g(\xi)} \frac{|d \xi|}{2 \pi}+\langle f, g\rangle-f(0) \overline{g(0)} \\
& =\frac{n}{\pi} \int_{\mathbb{T}}\left(1+P_{\lambda}(\xi)\right) f(\xi) \overline{g(\xi)} \frac{|d \xi|}{2 \pi}-f(0) \overline{g(0)}
\end{aligned}
$$

i.e.

$$
0=\frac{1}{\pi} \int_{\mathbb{T}}\left(1+P_{\lambda}(\xi)\right) f(\xi) \overline{g(\xi)} \frac{|d \xi|}{2 \pi}-\frac{f(0) \overline{g(0)}}{n} .
$$

Letting $n \rightarrow \infty$, we get

$$
\frac{1}{\pi} \int_{\mathbb{T}}\left(1+P_{\lambda}(\xi)\right) f(\xi) \overline{g(\xi)} \frac{|d \xi|}{2 \pi}=0 .
$$

It follows that

$$
f(0) \overline{g(0)}=0 .
$$

Without loss of generality, assume $f(0)=0$.

Since $\mathcal{D} \ominus \psi \mathcal{D}=\operatorname{span}\left\{1, K_{\lambda}\right\}$, there exist $c_{1}, c_{2}, c_{3}, c_{4} \in \mathbb{C}$ such that

$$
f=c_{1}+c_{2} K_{\lambda}, \quad g=c_{3}+c_{4} K_{\lambda} .
$$

By $f(0)=0$, we have $c_{1}+c_{2}=0$. Obviously, $c_{1} \neq 0$. Without loss of generality, assume $c_{1}=1$. Then

$$
f=1-K_{\lambda} .
$$

Since

$$
0=\langle f, g\rangle=\left\langle 1-K_{\lambda}, c_{3}+c_{4} K_{\lambda}\right\rangle=\overline{c_{4}}\left(1-K_{\lambda}(\lambda)\right)=\overline{c_{4}} \log \left(1-|\lambda|^{2}\right),
$$


we have $c_{4}=0$. Hence $g=c_{3}$, and obviously $c_{3} \neq 0$. But

$$
\begin{aligned}
& \frac{1}{\pi} \int_{\mathbb{T}}\left(1+P_{\lambda}(\xi)\right) f(\xi) \overline{g(\xi)} \frac{|d \xi|}{2 \pi} \\
= & \frac{\overline{c_{3}}}{\pi} \int_{\mathbb{T}}\left(1+P_{\lambda}(\xi)\right)\left(1-K_{\lambda}(\xi)\right) \frac{|d \xi|}{2 \pi} \\
= & \overline{c_{3}} \frac{1-K_{\lambda}(\lambda)}{\pi} \neq 0 .
\end{aligned}
$$

This contradicts (2.1). The above reasoning implies that $M_{\psi}$ has no proper reducing subspaces, completing the proof.

For the proof of Corollary 1.2, we need a lemma, which reads as follows:

Lemma 2.3. Let $\phi \in \mathcal{M}$. If $M_{\phi}$ is unitarily equivalent to $M_{z^{n}}$ for $n>0$, then $\phi$ is a Blaschke product of order $n$.

Proof. Let $U: \mathcal{D} \rightarrow \mathcal{D}$ be a unitary operator such that $U^{*} M_{\phi} U=M_{z^{n}}$, and let $I$ be the identity operator and $k_{\lambda}$ the normalization of $K_{\lambda}$ for $\lambda \in \mathbb{D}$, that is, $k_{\lambda}=K_{\lambda} /\left\|K_{\lambda}\right\|$.

It is easy to verify that $M_{z^{n}} M_{z^{n}}^{*}-I$ is compact and $k_{\lambda}$ weakly converges to 0 as $|\lambda| \rightarrow 1$. Hence, as $|\lambda| \rightarrow 1$,

$$
\left\langle M_{\phi} M_{\phi}^{*} k_{\lambda}, k_{\lambda}\right\rangle-1=\left\langle U\left(M_{z^{n}} M_{z^{n}}^{*}-I\right) U^{*} k_{\lambda}, k_{\lambda}\right\rangle \rightarrow 0
$$

Since

$$
\left\langle M_{\phi} M_{\phi}^{*} k_{\lambda}, k_{\lambda}\right\rangle-1=|\phi(\lambda)|^{2}-1
$$

this means that $|\phi(\lambda)|^{2} \rightarrow 1$ as $|\lambda| \rightarrow 1$. It follows that $\phi$ is an inner function. We claim that $\phi$ is a Blaschke product of finite order. Otherwise we can always find infinitely many $\lambda_{m} \in \mathbb{D}$ such that $\left|\lambda_{m}\right| \rightarrow 1$, but $\phi\left(\lambda_{m}\right) \rightarrow 0$. Since $z^{n}$ has order $n$, $\phi$ must has order $n$.

Proof of Corollary 1.2. The "if" part is obvious, so we give the proof only of the "only if" part.

Let $U: \mathcal{D} \rightarrow \mathcal{D}$ be a unitary operator such that $U^{*} M_{\phi} U=M_{z^{2}}$. By Lemma 2.3, $\phi$ must be a Blaschke product with two zeros $a, b \in \mathbb{D}$. Let $\phi=\varphi_{a} \varphi_{b}$.

Assume $a \neq b$. Then $\operatorname{ker} M_{\phi}^{*}=\operatorname{span}\left\{K_{a}, K_{b}\right\}$. Since $\operatorname{ker} M_{z^{2}}^{*}=\operatorname{span}\{1, z\}$, and $U\left(\operatorname{ker} M_{z^{2}}^{*}\right)=\operatorname{ker} M_{\phi}^{*}$, there exist $c_{1}, c_{2}, c_{3}, c_{4} \in \mathbb{C}$ such that

$$
U 1=c_{1} K_{a}+c_{2} K_{b}, \quad U z=c_{3} K_{a}+c_{4} K_{b}
$$

Let $e_{n}(z)=z^{n}$. Then

$$
U e_{2 n}=U M_{z^{2}} e_{2(n-1)}=M_{\phi} U e_{2(n-1)}=\cdots=M_{\phi}^{n} U 1=\phi^{n}\left(c_{1} K_{a}+c_{2} K_{b}\right) .
$$


By Carleson's integral formula,

$$
\begin{aligned}
& \left\langle U e_{2 n}, U e_{2 n}\right\rangle \\
= & \left\langle\phi^{n}\left(c_{1} K_{a}+c_{2} K_{b}\right), \phi^{n}\left(c_{1} K_{a}+c_{2} K_{b}\right)\right\rangle \\
= & \left|\phi(0)^{n}\left(c_{1}+c_{2}\right)\right|^{2}+D\left(\phi^{n}\left(c_{1} K_{a}+c_{2} K_{b}\right)\right) \\
= & \left|\phi(0)^{n}\left(c_{1}+c_{2}\right)\right|^{2}+\frac{n}{\pi} \int_{\mathbb{T}}\left(P_{a}(\xi)+P_{b}(\xi)\right)\left|c_{1} K_{a}(\xi)+c_{2} K_{b}(\xi)\right|^{2} \frac{|d \xi|}{2 \pi} \\
& +D\left(c_{1} K_{a}+c_{2} K_{b}\right) \\
= & \left|\phi(0)^{n}\left(c_{1}+c_{2}\right)\right|^{2}+\frac{n}{\pi} \int_{\mathbb{T}}\left(P_{a}(\xi)+P_{b}(\xi)\right)\left|c_{1} K_{a}(\xi)+c_{2} K_{b}(\xi)\right|^{2} \frac{|d \xi|}{2 \pi} \\
& +\left\langle c_{1} K_{a}+c_{2} K_{b}, c_{1} K_{a}+c_{2} K_{b}\right\rangle-\left|c_{1}+c_{2}\right|^{2} \\
= & \left(|\phi(0)|^{2 n}-1\right)\left|c_{1}+c_{2}\right|^{2}+1+\frac{n}{\pi} \int_{\mathbb{T}}\left(P_{a}(\xi)+P_{b}(\xi)\right)\left|c_{1} K_{a}(\xi)+c_{2} K_{b}(\xi)\right|^{2} \frac{|d \xi|}{2 \pi} .
\end{aligned}
$$

Hence for $n \geq 1$,

$$
\begin{aligned}
2 n= & \left\langle e_{2 n}, e_{2 n}\right\rangle=\left\langle U e_{2 n}, U e_{2 n}\right\rangle \\
= & \left(|\phi(0)|^{2 n}-1\right)\left|c_{1}+c_{2}\right|^{2}+1 \\
& +\frac{n}{\pi} \int_{\mathbb{T}}\left(P_{a}(\xi)+P_{b}(\xi)\right)\left|c_{1} K_{a}(\xi)+c_{2} K_{b}(\xi)\right|^{2} \frac{|d \xi|}{2 \pi} .
\end{aligned}
$$

It follows that

$$
\begin{aligned}
2= & \frac{\left(|\phi(0)|^{2 n}-1\right)\left|c_{1}+c_{2}\right|^{2}+1}{n} \\
& +\frac{1}{\pi} \int_{\mathbb{T}}\left(P_{a}(\xi)+P_{b}(\xi)\right)\left|c_{1} K_{a}(\xi)+c_{2} K_{b}(\xi)\right|^{2} \frac{|d \xi|}{2 \pi} .
\end{aligned}
$$

Letting $n \rightarrow \infty$, we have

$$
\frac{1}{\pi} \int_{\mathbb{T}}\left(P_{a}(\xi)+P_{b}(\xi)\right)\left|c_{1} K_{a}(\xi)+c_{2} K_{b}(\xi)\right|^{2} \frac{|d \xi|}{2 \pi}=2,
$$

and thus

$$
\left(|\phi(0)|^{2 n}-1\right)\left|c_{1}+c_{2}\right|^{2}+1=0 .
$$

Since $|\phi(0)|<1$, letting $n \rightarrow \infty$ gives

$$
\left|c_{1}+c_{2}\right|=1 \text {. }
$$

Then $|\phi(0)|^{2 n}=0$. So we must have $a=0$ or $b=0$.

By Theorem 1.1, if $U^{*} M_{\phi} U=M_{z^{2}}$, then $a+b=0$. So

$$
\phi(z)=\lambda z^{2}
$$

for some $\lambda$ with $|\lambda|=1$.

\section{REFERENCES}

1. A. Brown, On a class of operators, Proc. Amer. Math. Soc. 4(1953), 723-728. MR0059483 $(15: 538 \mathrm{c})$

2. L. Carleson, A representation formula for the Dirichlet integral, Math. Z. 73(1960), 190-196. MR 0112958 (22:3803)

3. C. Cowen. The commutant of an analytic Toeplitz operator, Trans. Amer. Math. Soc. 239(1978), 1-31. MR0482347(58:2420) 
4. K. Guo and H. Huang, On multiplication operators of the Bergman space: Similarity, unitary equivalence and reducing subspaces, to appear in J. Operator Theory.

5. K. Guo, S. Sun, D. Zheng and C. Zhong, Multiplication operators on the Bergman space via the Hardy space of the bidisk, to appear in J. Reine Angew. Math.

6. P. Halmos, Shifts on Hilbert spaces, J. Reine Angew. Math. 208(1961), 102-112. MR0152896 $(27: 2868)$

7. J. Hu, S. Shun, X. Xu and D. Yu, Reducing subspace of analytic operators on the Bergman space, Integr. Equ. Oper. Theory 49(2004), 387-395. MR2068435|(2005e:47073)

8. W. Ross, The classical Dirichlet space, Recent advances in operator-related function theory, Contemp. Math. 393, Amer. Math. Soc., Providence, RI, 2006, 171-197. MR2198379 (2006k:31007)

9. M. Stessin and K. Zhu, Reducing subspaces of weighted shift operators, Proc. Amer. Math. Soc. 130(2002), 2631-2639. MR.1900871 (2003c:47058)

10. S. Sun and Y. Wang, Reducing subspaces of certain analytic Toeplitz operators on the Bergman space, Northeast Math. J. 14(2)(1998), 147-158. MR1641059(99i:47047)

11. S. Sun and Y. Wang, The commutants of a class of analytic Toeplitz operators on Bergman spaces, Acta Sci. Natur. Univ. Jilinensis 2(1997), 4-8. MR.1604545 (98m:47034)

12. K. Zhu, Reducing subspaces for a class of multiplication operators, J. London Math. Soc. 62(2)(2000), 553-568. MR1783644 (2001h:47044)

School of Mathematics and Computer Science, Shanxi Normal University, Linfen, 041004, People's Republic of China

E-mail address: 1kzhao@sxnu.edu.cn 